\title{
Modelling and Control of a Hybrid Electric Propulsion System for Unmanned Aerial Vehicles
}

\author{
Ye Xie \\ School of Aerospace, Transport \\ and Manufacturing \\ Cranfield University \\ MK43 0AL, UK \\ 44-759-849-7446 \\ ye.xie@cranfield.ac.uk
}

\author{
Al Savvaris \\ School of Aerospace, Transport \\ and Manufacturing \\ Cranfield University \\ MK43 0AL, UK \\ 44-1234-754-626 \\ a.savvaris@cranfield.ac.uk
}

\author{
Antonios Tsourdos \\ School of Aerospace, Transport \\ and Manufacturing \\ Cranfield University \\ MK43 0AL, UK \\ 44-1234-758-578 \\ a.tsourdos@cranfield.ac.uk
}

\author{
Jason Laycock \\ Rotron Power Ltd \\ SP7 9AW, UK \\ 44-1747-440-510 \\ jason.laycock@rotron.co.uk
}

\author{
Andrew Farmer \\ Rotron Power Ltd \\ SP7 9AW, UK \\ 44-1747-440-515
}

andrew.farmer@giloindustriesgroup.com

\begin{abstract}
This paper presents the modelling and control of a hybrid electric propulsion system designed for unmanned aerial vehicles. The work is carried out as part of the AIRSTART project in collaboration with Rotron Power Ltd. Firstly, the entire parallel hybrid powertrain is divided into two powertrains to facilitate the modelling and control. Following this, an engine model is built to predict the dynamics between the throttle request and the resulting output. It is then validated by comparing with experimental data. On the basis of $d-q$ model of the motor/generator, a good estimation of torque loss at steady state is achieved using the efficiency map. Next, a rule-based controller is designed to achieve the best fuel consumption by regulating the engine to operating around its ideal operating line. Following the integration of the models and controller, the component behaviour and control logic are verified via the final simulation. By enabling the engine to operate at its best fuel economy condition, the hybrid propulsion system developed in this research can save at least $7 \%$ on fuel consumption when compared with an internal combustion engine powered aircraft.
\end{abstract}

\section{TABle of Contents}

1. INTRODUCTION

2. HYBRID CONFIGURATION

3. MODELLING AND CONTROL OF HYBRID ELECTRIC PROPULSION SYSTEM 3

4. RESULTS AND ANALYSIS 9

5. CONCLUSION

REFERENCES.

\section{INTRODUCTION}

Nowadays, a growing number of Unmanned Aerial Vehicles (UAVs) are powered by electric motors (EMs), because of lower emissions and noise, better overall efficiency, and lower maintenance requirements. However, the specific energy of electric energy storage sources, e.g. batteries, is much lower than that of fossil fuel [1]. As a result, the internal combustion engine (ICE) is preferred for relatively large or long-endurance UAVs, due to its high power and energy density [2]. The hybrid electric propulsion system (HEPS) combines an electric powertrain with a conventional combustion engine to provide propulsion, in other words, being able to have the energy efficiency of an electric propulsion system with the extended range of an ICE. The aforementioned benefits make HEPS an attractive option to explore for powering UAVs.

The Air Force Institute of Technology (AFIT) has conducted several studies on hybrid electric technologies. Harmon et al. began the project with the design of neural network control for a parallel HEP [3]. Continuing on from this work, Ausserer [4] and Molesworth [5] implemented the physical integration and validation of a prototype, continuing the theoretical studies, which were started by Harmon. Queensland University of Technology (QUT) also conducted some studies [6-7]. Glassock et al. successfully downsized the engine and improved the overall propulsive efficiency compared to the non-hybrid system. University of Colorado [7] achieved an increase in fuel efficiency over traditional gas-powered aircrafts, by utilizing a clutch-less gearing system in parallel configuration. Bental Industries [8] announced a type of "hybrid" engine, combined with an EM acting as a starter for the ICE and also as an alternator for the battery. In 2010, HEPS solution for general aviation was developed by the German aircraft manufacturer - Flight Design [9].Three years later, the first pilot-operated series hybrid electric airplane (DA36 E-Star) developed by Siemens, completed its debut flight on June 8, 2011 [10].

HEPS can provide better fuel economy and lower emissions without compromising performance. In addition, it can provide on-board electrical regeneration for powering 
different systems, but this flexibility and diversity comes at a cost of increase complexity in the modelling and control.

The mean value model (MVM) [11] is widely used for modelling the engine, because it can simplify phenomena that are too fast or complex. However, some details in MVM, such as inlet manifold dynamics, increase the complexity and cost of modelling. Consequently, a modelling approach that approximates the engine torque response with a first-order or second-order transfer function [12] is largely introduced. This approach further simplifies the modelling but it cannot reveal the relationship between the throttle position and output capability. The first part of this paper presents a model which can estimate the performance of engine control unit (ECU) and link a throttle command with the torque output.

Similar modelling issue also arises when the first or second order system is used for motor/generator modelling [13]. This modelling method prevents unnecessary complexity but it does not cover the electromagnetic characteristics of motor/generator, which are necessary for deriving required voltage and current. The well-established $d-q$ model used to model motor/generator [14] is also used in the paper, but focuses on the estimation of energy losses and the integration of different control loops and inverter/rectifier.

Unlike conventional single-source-powered vehicle, the controller in an HEPS is normally comprised of supervisory controller and low-level component controllers. The supervisory controller functions at the energy management level, splitting power or torque requests between the ICE and electric powertrain [15]. Broadly, it can be classified into rule-based [16][17] and optimization-based approaches [18]. The rule-based control is made up a series of logic statements and the transition from one mode to another depends on the predefined criteria. Hence, it is reliable, robust and easier to implement when compared with the optimization algorithm approach. As a first step, a rule-based control is developed in this paper to optimize the engine behaviour based on the fuel consumption criterion.

The results presented in this paper are part of the ongoing work performed as part of the AIRSTART project. AIRSTART is a $£ 3.2$ million collaborative Research and Development project developing key technologies to support routine small Unmanned Aerial Vehicle (UAV) operations Beyond Visual Line of Sight (BVLOS). Cranfield is working on the hybrid propulsion system, converting a Rotron UAV engine into a hybrid combustion-electric system. The platform for testing the hybrid propulsion system is a remotely piloted multi-purpose UAV - the Aegis. First, the high-level system architecture of the parallel hybrid powertrain is presented, containing the two powertrains. Next, the main components are modelled for example: an engine model is built to represent the dynamics between the throttle request and the resulting output, while the ECU is modelled as a function derived from the experimental-data. The torque loss of motor/generator is estimated using the efficiency map from the manufacturer, which is followed by the development of a three-loop control strategy. The gear ratio is selected based on the analysis of engine fuel map and motor/generator efficiency map. Lastly, the energy management strategy is governed by a series of logics that can enable the engine to operate in the minimum fuel consumption regime. This rule-based controller is also in charge of switching the control mode of motor/generator and the operating mode of the entire HEPS.

The engine model is validated by comparing the simulation results with the experimental data. Finally, the complete HEPS, including the different operating modes is tested in simulation to assess the performance of the models and controller.

\section{HYBRID CONFIGURATION}

In a hybrid propulsion system, two or more power sources are combined together to increase the efficiency of the vehicle. There are various hybrid powertrain configurations currently in use. The most commonly used configurations are series, parallel, and series-parallel architecture. Among them, the parallel configuration permits a smaller engine or motor to be used as compared to the series one. The parallel architecture for this configuration is also lighter. In addition, to having the ability to select in which mode to operate e.g.

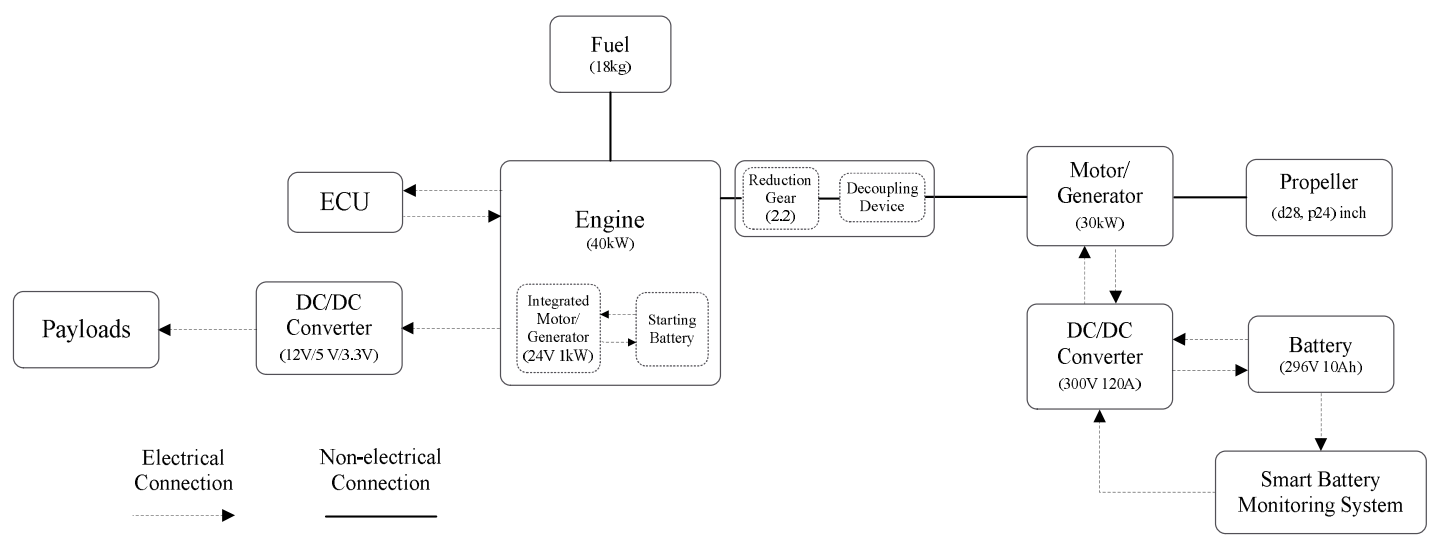

Figure 1 - Complete Parallel hybrid propulsion system 
electric, combined mode or internal combustion engine mode only. According to [19] and [20], the parallel configuration is best suited for long-endurance UAVs.

The high-level parallel hybrid propulsion system developed in this project is shown in Figure 1. An ECU is used for engine control; and the engine is started using the integrated motor and a dedicated battery. Once the engine started, the integrated motor can also act as a generator to power the aircraft systems (avionics, flight controller, actuators etc.) and the payload via a DC/DC converter. On the other side, the output shaft is connected to the motor/generator through a decoupling device and a reduction gear. An intelligent battery monitoring system monitors the charging/discharging of the battery.

The generator charges the battery if the state-of-charge (SOC) is low. This setup enables the engine to operate in the highest efficiency region with the motor/generator providing the additional power required and also the ability to provide instant power to cover rapid transient demands in power. The proposed energy management strategy can optimize the amount of motor/generator power and the time depending on the operating conditions, the SOC, and the flight mission. Moreover, this architecture also permits the motor, in the event of an engine failure or 'stealth mode', to act as the primary powerplant and drive the propeller.

The engine, motor/generator and battery have a significant impact on the steady-state performance and even the dynamics of the complete HEPS. Hence these three components are modelled in detail in this study as shown in Figure 2. The remaining parts can be simplified to facilitate the analysis of features of the whole system. The energy flow and components of the two powertrains are shown in Figure 2. The red line represents the fuel flow to the engine and the corresponding mechanical torque/power produced, while the electric power supports the driver via the blue line. Hereafter, for the analysis carried out in this paper the modelling and control are both based on this simplified architecture.

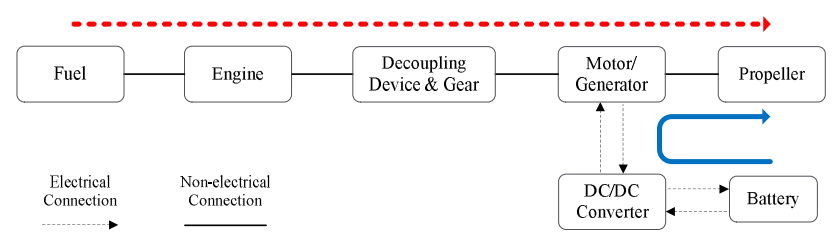

Figure 2 - Energy flows of combustion powertrain and electric powertrain

\section{Modelling AND CONTROL OF HYBRID Electric Propulsion System}

\section{A. Modelling}

This section presents the modelling of the engine, motor/generator and battery in detail, while all mechanical components (e.g. gear and decoupling device) and the DC/DC converter are simplified. With regard to the propeller, the aerodynamic performance is estimated using the actuator disc theory [21-22].

1) Engine-A simplified mean value engine model that does not include manifold dynamics and flow rate dynamics, is used to represent the dynamics between the throttle request and the resulting crankshaft speed of the rotary engine. The engine torque request is calculated as a function of the throttle, engine speed and make-up from the idle speed controller:

$$
T_{\text {ice,ref }}=f\left(\delta_{\text {ice }}, \omega_{\text {ice }}\right)+T_{\text {ice, idle }} .
$$

Here, $T_{\text {ice,ref }}$ is the torque request and $T_{\text {ice,idle }}$ is the idle torque. $f\left(\delta_{\text {ice }}, \omega_{\text {ice }}\right)$ is an experimental-data based function which realizes the utility of the ECU. That implies that $\delta_{i c e}$ used here is a virtual throttle command, which is generated by the engine speed controller. The speed controller of engine is modelled as a PI controller, the same as the idlespeed controller. It is worth noting that $f\left(\delta_{\text {ice }}, \omega_{\text {ice }}\right)$ already includes the engine loss. Therefore, there is no need to estimate that.

The relationship between the engine torque request and the induced torque is given by:

$\dot{T}_{i c e}(t)=-\frac{1}{\tau_{i c e}} T_{i c e}(t)+\frac{1}{\tau_{i c e}} T_{i c e, r e f}\left(t-\delta_{i c e, p r c}\right)$,

where $T_{i c e}$ is the induced engine torque and $\tau_{i c e}$ is the engine time constant due to fuel/air injection. $\delta_{\text {ice }, p r c}=$ $\vartheta_{\text {rotary }} / \omega_{\text {ice }}$ stands for the time for one engine process, namely the power stroke to induction transport. Since the working principle of the rotary engine is different from the piston engine, this process time needs to be changed according to the characteristics of the rotary engine.

When the engine is decoupled from the generator, its rotational dynamics is governed by $\frac{d \omega_{i c e}}{d t}=T_{i c e} / J_{i c e}$. While for the combustion powertrain, since the rotational dynamics of engine and generator are coupled, the explanation in detail is covered section 3.2) of this paper.

Table 1 - RT300 engine parameters

\begin{tabular}{|c|c|c|}
\hline Parameters & Symbols & RT300 \\
\hline Maximum speed (rpm) & - & 7500 \\
Idle speed (rpm) & - & 2000 \\
Time constant (sec) & $\tau_{\text {ice }}$ & 0.002 \\
Angle for one process (rad) & $\vartheta_{\text {rotary }}$ & $\pi / 2$ \\
Moment of inertia $\left(\mathrm{kg}^{*} \mathrm{~m}^{2}\right)$ & $J_{\text {ice }}$ & 0.02425 \\
\hline
\end{tabular}


The parameter values required for the modelling are shown in Table 1.

Meanwhile, the engine fuel consumption rate $\dot{m}_{f u e l}$ is calculated using the static map, which is a function of the torque and engine speed (see Figure 3). This approach has been proven to give a satisfactory prediction of fuel consumption under different operating conditions [23-24]. The brake specific fuel consumption (BSFC) values are used here to emphasize the trend of fuel consumption per KW. As shown in Figure 3, the engine points better fuel economy per $\mathrm{KW}$ at the higher speeds. It is recommended that the engine operates in the range of 4500-5500 rpm, in order to extend the lifecycle of engine while maintaining good fuel performance.

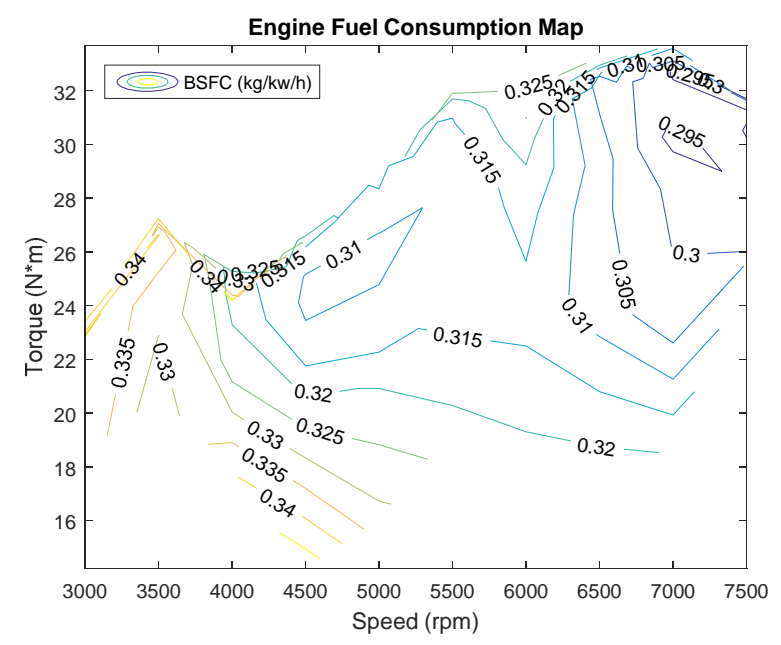

Figure 3 - Fuel consumption map of ICE

\section{2) Motor/Generator-}

a) Motor: The EMRAX228 is a permanent magnet synchronous motor/generator (PMSM/PMSG). In the present work, the $d-q$ frame is introduced to force all sinusoidally varying inductances to become constants. Under some assumptions [25], the dynamic behaviour of the three-phase PMSM can be described by the differential equations, in the rotor $d-q$ reference frame [26], as follows:

$$
\begin{gathered}
\frac{d i_{d}}{d t}=\left(v_{d}-R i_{d}+\omega_{s} L_{q} i_{q}\right) / L_{d}, \\
\frac{d i_{q}}{d t}=\left(v_{q}-R i_{q}-\omega_{s} L_{d} i_{d}-\omega_{s} \lambda_{m}\right) / L_{q},
\end{gathered}
$$

where $v_{d}$ and $v_{q}$ are the $d$ - and $q$ - axis stator voltages, whereas $i_{d}$ and $i_{q}$ are the $d$ - and $q$ - axis stator currents. $L_{d}$ and $L_{q}$ are the $d$ - and $q$ - axis stator inductances, respectively. $\lambda_{d}$ and $\lambda_{q}$ are the $d$ - and $q$-axis stator flux linkages, while $\lambda_{m}$ is the magnet mutual flux linkage. $R$ represents the stator resistance and $\omega_{s}$ is the inverter frequency.
Without considering the inertia of the output shaft, the equation of the motor dynamics, namely the rotational dynamics of the electric powertrain is:

$$
\frac{d \omega_{m o t}}{d t}=\left(T_{m o t}-T_{m o t, l o s s}-T_{p r o p}\right) / J_{m o t},
$$

in which $\omega_{m o t}$ is the rotor speed, $J_{m o t}$ is the moment of inertia, and $T_{\text {prop }}$ is the load torque caused by the rotating of the propeller. The electromagnetic torque $\left(T_{m o t}\right)$ produced by the motor is given in [26]:

$$
T_{m o t}=\frac{3 p\left[\left(\lambda_{m} i_{q}+\left(L_{d}-L_{q}\right) i_{d} i_{q}\right)\right]}{2}=\frac{3}{2} p \lambda_{m} i_{q},
$$

where $p$ is the number of pole pairs and the inverter frequency is related to the rotor speed with $\omega_{s}=p \omega_{m o t}$. It should be noted that the right hand side of equation (6) is obtained by forcing $i_{d}$ to zero. Thus, $i_{q}$ can be represented as $\frac{2 T_{m o t}}{3 p \lambda_{m}}$.

On the other hand, the motor torque loss $\left(T_{\text {mot,loss }}\right)$ including friction loss and damping is approximated by the efficiency map from the manufacturer (see Figure 4). The efficiency $\eta$ is defined as:

$$
\eta=\frac{P_{\text {out }}}{P_{\text {in }}}=\frac{T_{\text {prop }} \omega_{\text {mot }}}{P_{\text {in }}}
$$

in which $P_{\text {in }}$ stands for the total electromagnetic power and is expressed by [27]:

$$
P_{\text {in }}=3\left(v_{d} i_{d}+v_{q} i_{q}\right) / 2
$$

According to mechanical characteristics under equilibrium conditions, the torque loss acting on the rotor is:

$$
T_{m o t, l o s s}=T_{m o t}-T_{\text {prop }}
$$

By substituting equations (6)-(9), a quadratic equation of $T_{\text {mot,loss }}$ can be addressed:

$$
\begin{gathered}
k T_{\text {mot }, \text { loss }}^{2}+\left(\omega_{\text {mot }}+2 k T_{\text {prop }}\right) T_{\text {mot }, \text { loss }}+ \\
k T_{\text {prop }}^{2}+\left(1-\frac{1}{\eta}\right) \omega_{\text {mot }} T_{\text {prop }}=0,
\end{gathered}
$$

where $k=\frac{2 R}{3 \lambda_{m}^{2} p^{2}}$. Different from the study in [27], since the torque loss is apparently related to other variables (see Figure 5), it is not approximated by the function that is only dependent on rotor speed. The torque loss is given as a function of electromagnetic torque and rotor speed so as to integrate the loss into the $d-q$ model. An algebraic representation will be studied in future work. 


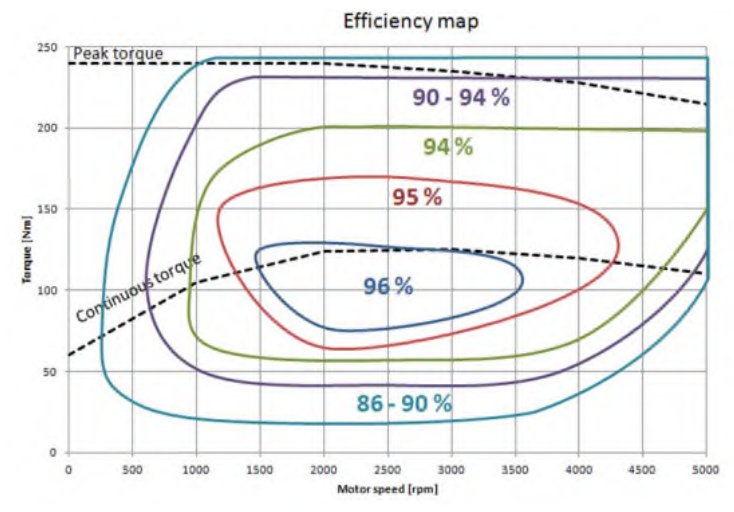

Figure 4 - Efficiency map of PMSM

b) Generator: The electrical dynamics of the PMSG is the same as one of the PMSM. To distinguish between charging and discharging process, the reversal of the flow of current and voltage is reflected by changing the corresponding variables' signs. That is:

$$
\begin{gathered}
\frac{d i_{d}}{d t}=\left(-v_{d}-R i_{d}+\omega_{s} L_{q} i_{q}\right) / L_{d}, \\
\frac{d i_{q}}{d t}=\left(-v_{q}-R i_{q}-\omega_{s} L_{d} i_{d}+\omega_{s} \lambda_{m}\right) / L_{q} .
\end{gathered}
$$

Please note that, with respect to the combustion powertrain, the rotational dynamics of engine is coupled into the generator. Thus, the ICE torque and its inertia need to be transferred into the coordinate of the PMSG. Without considering the inertia of rotating shaft and propeller, the rotational dynamics of the generator is governed by the following equation:

$$
\frac{d \omega_{g e n}}{d t}=\frac{G T_{i c e}-\left(T_{\text {gen }}+T_{\text {gen,loss }}+T_{\text {prop }}\right)}{G^{2} J_{i c e}+J_{\text {gen }}},
$$

in which $\omega_{\text {gen }}$ is the generator rotor speed. $T_{i c e}$ is the mechanical torque applied by the ICE, whereas $T_{\text {prop }}$ is the load torque caused by the propeller. $J_{i c e}$ and $J_{\text {gen }}$ are the inertia of the engine and generator, respectively. The electromagnetic torque $\left(T_{g e n}\right)$ and power $\left(P_{\text {out }}\right)$ of generator are:

$$
\begin{gathered}
T_{g e n}=\frac{3}{2} p \lambda_{m} i_{q}, \\
\text { and } P_{\text {out }}=3\left(v_{d} i_{d}+v_{q} i_{q}\right) / 2,
\end{gathered}
$$

if the vector control is applied. Likewise, the generator torque loss $\left(T_{\text {gen,loss }}\right)$ is related to driving torque $T_{\text {drive }}$ with:

$$
T_{\text {gen,loss }}=T_{\text {drive }}-T_{\text {gen }}-T_{\text {prop }} .
$$

The efficiency is given by:

$$
\eta=\frac{P_{\text {out }}}{P_{\text {in }}}=\frac{P_{\text {out }}}{T_{\text {drive }} \omega_{\text {gen }}} .
$$
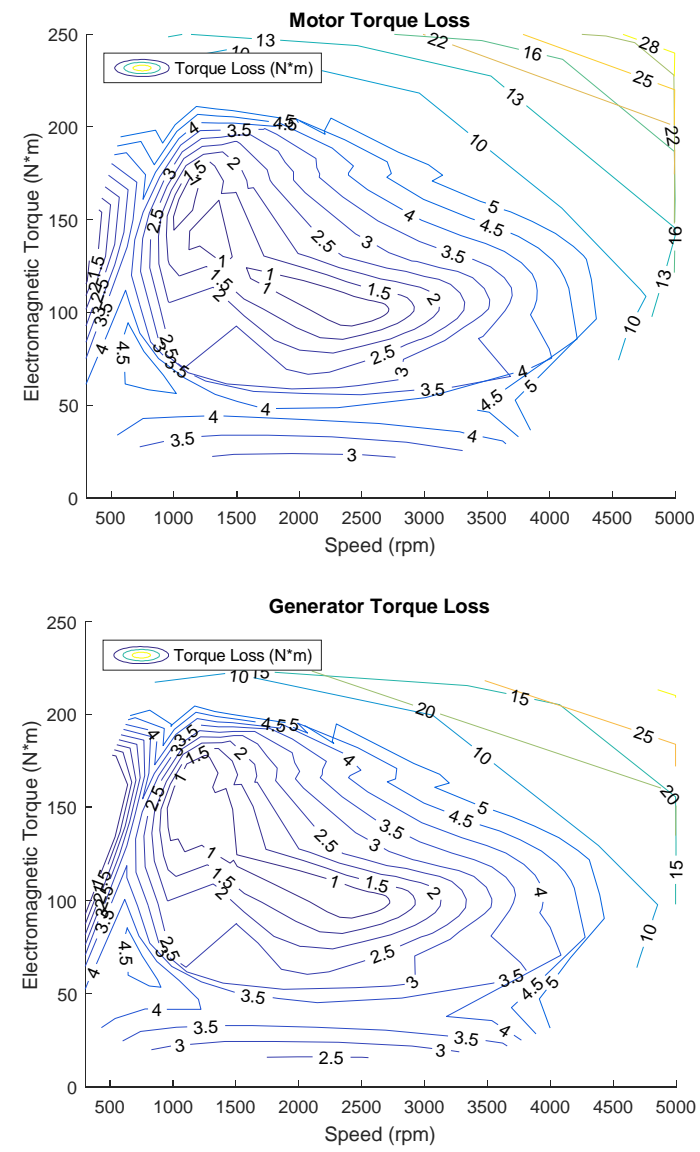

Figure 5 - Torque loss of PMSM/PMSG

Table 2 - EMRAX228 parameters

\begin{tabular}{|c|c|c|}
\hline Parameters & Symbols & EMRAX228 \\
\hline Maximum speed $(\mathrm{rpm})$ & - & 5000 \\
D-axis stator inductance $(\mu \mathrm{H})$ & $L_{d}$ & 175 \\
Q-axis stator inductance $(\mu \mathrm{H})$ & $L_{q}$ & 180 \\
Stator resistance $(\Omega)$ & $R$ & 0.018 \\
Magnet mutual flux $(\mathrm{Wb})$ & $\lambda_{m}$ & 0.0542 \\
Moment of inertia $\left(\mathrm{kg}^{*} \mathrm{~m}^{2}\right)$ & $J_{m o t} / J_{g e n}$ & 0.0421 \\
Number of pole pairs & $p$ & 10 \\
\hline
\end{tabular}

Following the similar deduction of $T_{\text {mot,loss }}$, a quadratic equation of $T_{\text {gen,loss }}$ can also be addressed. Consequently, the loss estimation of motor and generator are both shown in Figure 5. It is obvious that the torque loss develops quickly when the speed is above $4500 \mathrm{rpm}$ or the torque is higher than $200 \mathrm{~N} * \mathrm{~m}$ for both motor and generator mode. Besides, the torque loss increases with the increase of torque when the torque is under $50 \mathrm{~N} * \mathrm{~m}$, while the variation of speed have no large influence on the torque loss. More importantly, the 
minimum loss of the motor is around $1000-3000 \mathrm{rpm}$ and 80-180 $\mathrm{N}^{*} \mathrm{~m}$. These characteristics are the same for the generator.

The parameters of PMSM/PMSG required for the modelling are shown in Table 2.

c) Control: The control of PMSM/PMSG can be separated into three loops (see Figure 6): vector control, torque control and speed control. All control loops are realized as PI controllers. To convert AC motors into equivalent DC motors which is separately excited, the vector control is used to recreate the orthogonal flux by controlling $i_{q}$ and $i_{d}$, respectively [25]. Then, the torque following is achieved by forcing $i_{d}$ to zero and regulating $i_{q}$. Similarly, the required speed is obtained by controlling the torque. This control structure can accept both speed and torque references. Meanwhile, the transition between two control modes is governed by the supervisory control.

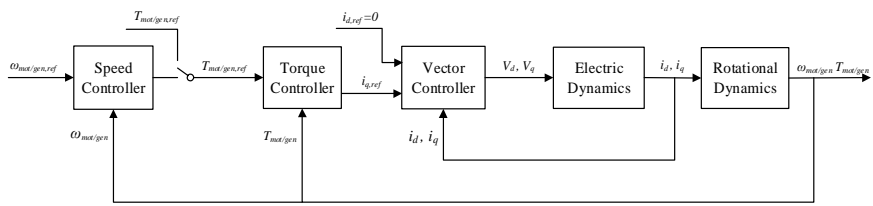

Figure 6 - Diagram of PMSM/PMSG control loops

d) Inverter/Rectifier: The average model of the voltage source converter is described by applying the input-output electrical relationships of the average pulse-widthmodulated (PWM) converter in the $d$ - $q$ frame [28]:

$$
\begin{gathered}
{\left[\begin{array}{l}
v_{d} \\
v_{q}
\end{array}\right]=\left[\begin{array}{l}
d_{d} \\
d_{q}
\end{array}\right] V_{d c} ;} \\
I_{d c}=\frac{3}{2}\left[\begin{array}{ll}
i_{d} & i_{q}
\end{array}\right]\left[\begin{array}{l}
d_{d} \\
d_{q}
\end{array}\right],
\end{gathered}
$$

in which $V_{d c} / I_{d c}$ is the DC voltage/current drawn by the inverter/rectifier from the DC/DC converter, and $d_{d}, d_{q}$ are continuous duty cycle functions in the $d$ - and $q$ - axis, respectively. The instantaneous values of $d_{d}$ and $d_{q}$ can be obtained from the actual values of $V_{d c}, v_{d}$ and $v_{q}$.

3) Battery-There are basically three types of battery models reported in the existing literature: experimental, electrochemical and electric-circuit based. The main purpose of battery modelling is to present its charge/discharge characteristics and predict the SOC which is important for the energy management. Therefore, experimental models seem inadequate while electrochemical models are too complex. An electric-circuit battery model based on the work presented in [29] is used. This model follows the approach proposed by Shepherd in 1965 [30], which neglects the faster RC circuit dynamics and pays attention to the open circuit voltage and current. The discharging/charging characteristics and SOC definition of lithium-ion polymer (LiPo) batteries are governed by (20) (22).

$$
\begin{gathered}
E=E_{0}-K \cdot \frac{Q}{Q-Q_{b a t t, c}} \cdot Q_{b a t t, c}-K . \\
\frac{Q}{Q-Q_{b a t t, c}} \cdot i^{*}+A e^{-B \cdot Q_{b a t t, c}} ; \\
E=E_{0}-K \cdot \frac{Q}{Q-Q_{b a t t, c}} \cdot Q_{b a t t, c}-K \cdot \frac{Q}{Q_{b a t t, c}-0.1 Q} \\
i^{*}+A e^{-B \cdot Q_{b a t t, c}} ; \\
\operatorname{SOC}=\frac{Q-Q_{b a t t, c}}{Q},
\end{gathered}
$$

where $E$ is the battery open circuit voltage, $E_{0}$ is the battery constant voltage, $i^{*}$ is the filtered current obtained by a lowpass filter, $Q_{b a t t, c}=\int i_{b a t t} d t$ is actual battery charge, $K$ is the polarization resistance $(\Omega), Q$ is the battery capacity, $A$ is the exponential voltage coefficient $(\mathrm{V}), B$ is the exponential capacity coefficient $\left((\mathrm{Ah})^{-1}\right)$. The filtered current $i^{*}$ is used to reproduce a slow dynamic behaviour of voltage for a current step response, which is more compatible with the experimental results [29]. Finally, the output voltage $V_{b a t t}$ is obtained by $V_{\text {batt }}=E-i_{\text {batt }} \cdot R_{\text {int }}$, in which $i_{\text {batt }}$ is the output current and $\mathrm{R}_{\text {int }}$ represent the internal resistance.

The parameters of LiPo battery required for the modelling are shown in Table 3.

Table 3 - Battery parameters

\begin{tabular}{|c|c|c|}
\hline Parameters & Symbols & LiPo \\
\hline Capacity $(\mathrm{Ah})$ & $Q$ & 10 \\
Internal resistance $(\Omega)$ & $R_{\text {int }}$ & 0.296 \\
Constant voltage $(\mathrm{V})$ & $E_{0}$ & 320.6795 \\
$\begin{array}{c}\text { Polarization constant or } \\
\text { resistance }(\Omega)\end{array}$ & $K$ & 0.16709 \\
$\begin{array}{c}\text { Exponential voltage } \\
\text { coefficient }(\mathrm{V})\end{array}$ & $A$ & 25.1477 \\
$\begin{array}{c}\text { Exponential capacity } \\
\text { coefficient }((\text { Ah) }\end{array}$
\end{tabular}

\section{B. Rule-Based Control}

The main function of supervisory controller of the hybrid propulsion system is to improve the high-level vehicle performance, e.g. fuel economy and powertrain efficiency, covering the complete flight mission. In this part of the investigation, only the engine fuel consumption is taken into consideration. Therefore, the engine Idea Operating Line (IOL) with a rule-based control is introduced in this study. The IOL is an optimum condition at which the fuel consumption per KW is minimal at steady state [31]. Thus, operating at the IOL can achieve the least fuel consumption of the engine. A rule-based controller, essentially, is a set of rules that determines which driver is suitable to use and how 
much power applied in the engine and motor/generator, separately. Therefore, it can ensure that the engine operates at its fuel economy optimum point by using IOL.

1) Ideal Operating Line-Typically, the fuel consumption map and the power map are required to determine the IOL for an engine. Next, the power map is overlapped on the fuel map and then points with the lowest fuel consumption can be calculated. Those points constitute the IOL and give the value of BSFC, fuel consumption, torque, and power at the IOL (see Figure 7).
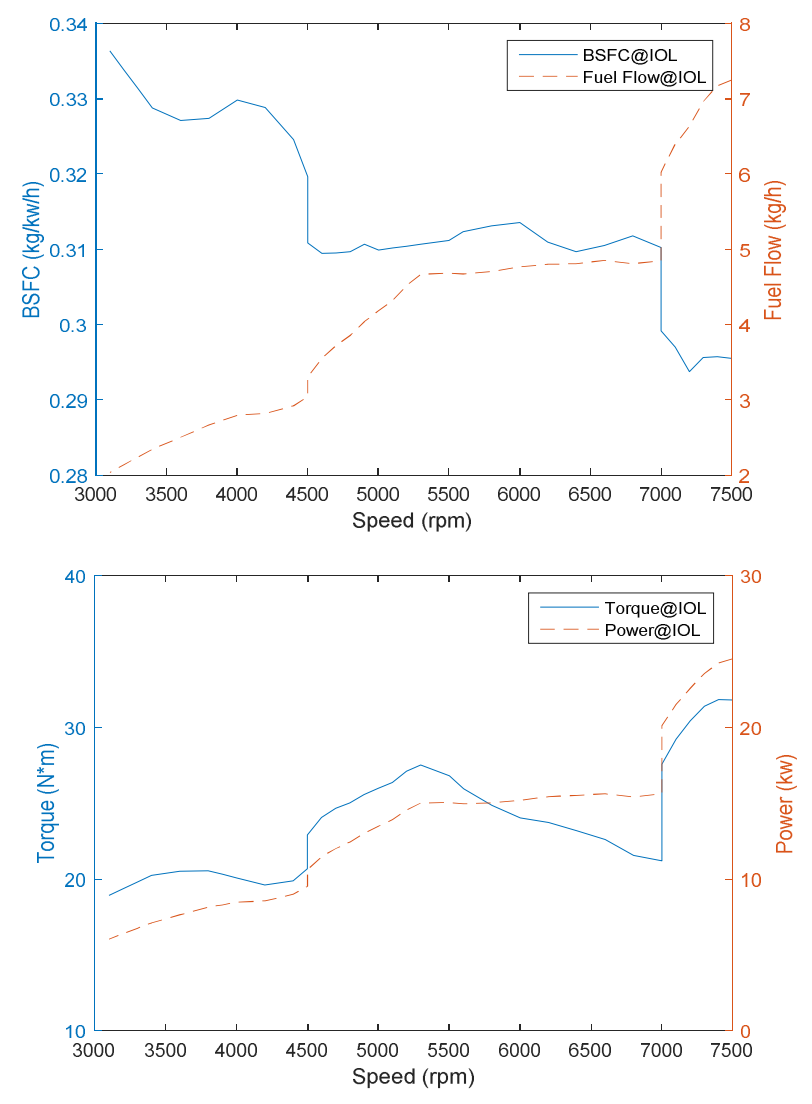

Figure 7 - Fuel consumption, torque, and power at ideal operating line of ICE

As shown in the Figure 7, the optimum BSFC decreases with the increasing of the speed and experiences a steady phase between 4500-7000 rpm; while the real fuel flow has an opposite trend. These imply that RT300 has higher fuel efficiency at the higher speeds when runs at the minimum fuel consumption condition. Also, the engine reaches a relatively high torque around $5400 \mathrm{rpm}$ and $7500 \mathrm{rpm}$. In addition, a rapid change occurs roughly at $7000 \mathrm{rpm}$ for all variations.

2) Control Strategy-The control strategy developed in this project takes the power requirement and SOC as the main decision variables. There are four main operating modes in this rule-based control scheme: Electric Mode, Fuel Mode, Charge Mode and Combined Mode.

(1) The first is the Electric Mode, it is engaged when there is an engine failure or the 'stealth flight mode' is required. In this mode, the motor is the main driver and the engine is disengaged from the power train. The motor is powered by the energy stored in the battery;

(2) The second is the Fuel Mode, in which the aircraft is driven by the engine and the battery is not being charged. Put differently, the SOC of the battery is at a high level and no charging is required;

(3) The third is the Charge Mode, it is similar to the Fuel Mode but in this mode the motor/generator operates as a generator to charge the battery. The Charge Mode has two divisions: Extra Charge Mode and Regular Charge Mode it depends on the availability of extra power. When the power request of the engine is lower than the corresponding power at IOL, the controller will select the Extra Charge Mode. On the other hand, the Regular Charge Mode is selected when the SOC of battery is under a certain level. Besides, in the Regular Charge Mode, the power required to charge is managed by the algorithm taking into consideration SOC as the main decision variable. Thus, the current is relatively high and even higher at the lower SOC;

(4) Finally, the Combined Mode is developed to handle the situation where the engine or motor cannot meet the power demands separately. This mode can be also used to enable the engine to operate at the minimum fuel consumption state by combining motor power.

The flow chart in Figure 8 illustrates the rules logic to switch between the different operating modes. If the engine fails, the Electric Mode is selected. If the power requirement is higher than the maximum power of engine or motor, the HEPS enters the Combined Mode; otherwise, the supervisory controller will compare the power requirement with the IOL power if the 'stealth flight' is not requested it will enter the Charge Mode. The Charge Mode is separated into Extra Charge Mode and Regular Charge Mode. If none of above three modes is feasible, then the HEPS will enter into the Fuel Model. 


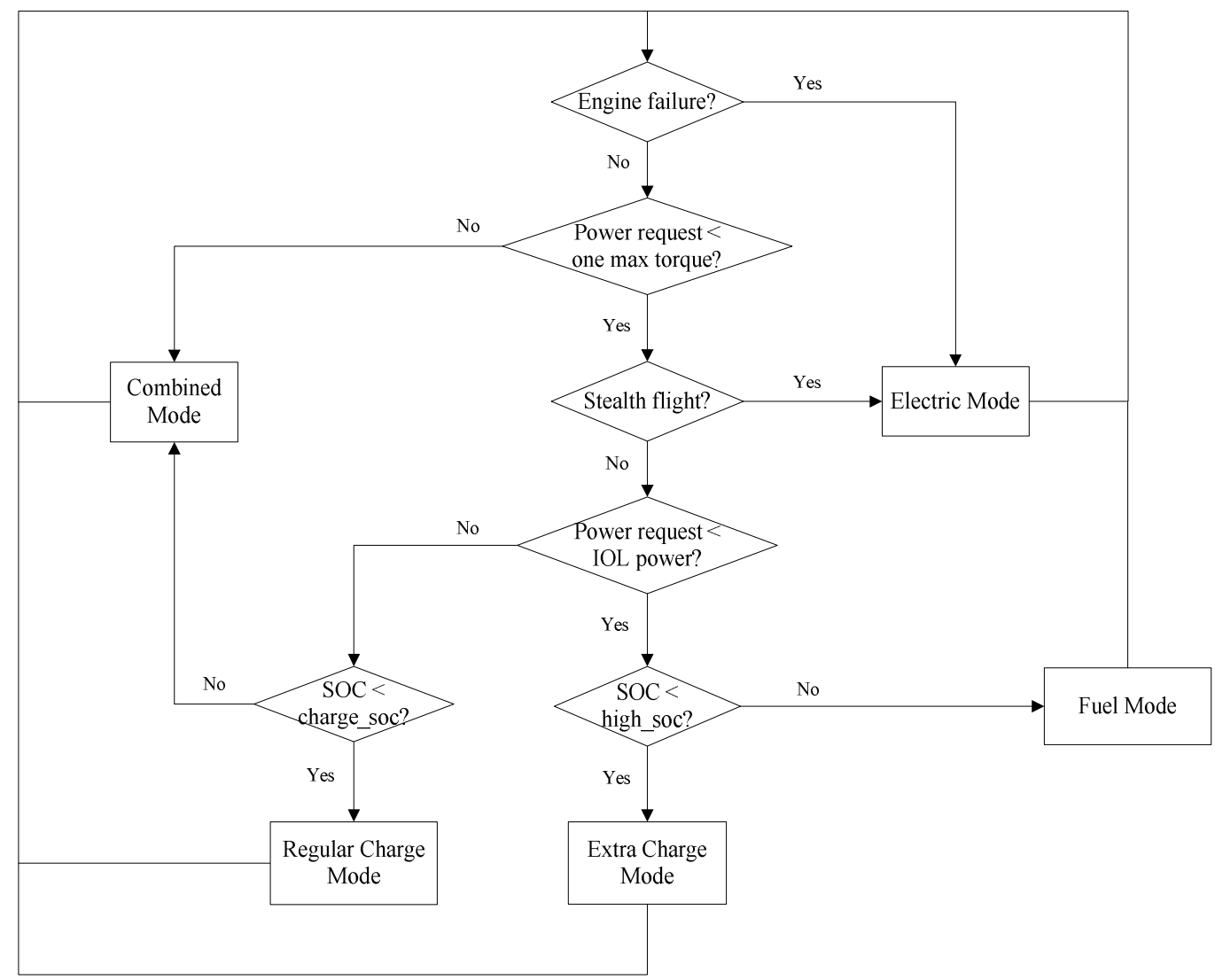

Figure 8 - Flow chart of rule-based control strategy

\section{Integration of Models and Supervisory Control}

Typically, the complete parallel HEPS constitutes components models and the supervisory controller (see Figure 9). The coupling device is modelled as a switch, which is responsible for the transition between combustion powertrain and electric powertrain. As mentioned previously, the optimal speed range of the engine and motor/generator is around 4500-5500 rpm and 1000-3000 $\mathrm{rpm}$, respectively. To obtain the best match when the engine and motor/generator operate together, the ratio of reduction gear is configured to 2.2 . With regards to the DC/DC converter, only the efficiency of the power conversion is taken into consideration.
Figure 9 illustrates the signal flow between different model blocks and controller, where the direction of the arrow implies the decision-making of one signal (variation). In other words, the block (models or controller) where the arrow starts from is the device to determine the values of variations. Here, the control signals from the supervisory controller are represented by dash lines, while dotted lines denote variations fed back to the supervisory controller.

The power requirement $\left(P_{\text {req }}\right)$ is dependent on the propeller torque $\left(T_{\text {prop }}\right)$ and power requirement from the payloads (for our system it is estimated to be around $500 \mathrm{~W}$ ). Following this, the rule-based controller decides the operating condition $\left(\right.$ flag $\left._{\text {mode }}\right)$ and speed or torque reference of the engine and

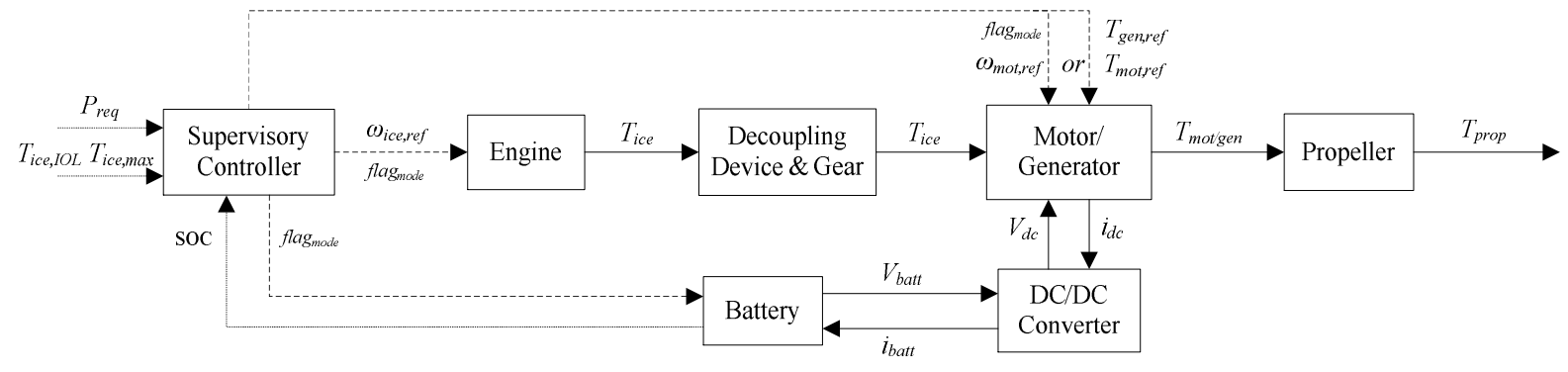

Figure 9 - Block diagram of hybrid propulsion system 
motor/generator, according to $P_{\text {req }}$ from the aircraft and the SOC of the battery. The engine torque then transits to the motor/generator via mechanical coupling devices. Meanwhile, the motor/generator selects its control mode (torque or speed control) and operating mode (motor or generator) and finally output the torque to the propeller. The discharge/charging process is also managed by the supervisory controller.

\section{RESULTS AND ANALYSIS}

\section{A. Validation of Engine Model}

By coupling the engine and motor, the motor/generator can work as a generator and the extra power produced can be used to charge the battery when its SOC is low. The results in Figure 10a show the speed tracking performance of the engine when it is driving the propeller. The speed tracking function is performed by the ECU. As shown in the graph, the engine follows the reference speed $(5000 \mathrm{rpm})$ profile from the initial speed $(4000 \mathrm{rpm})$ within a reasonable rising time (around $3 \mathrm{sec}$ ) and without overshooting.

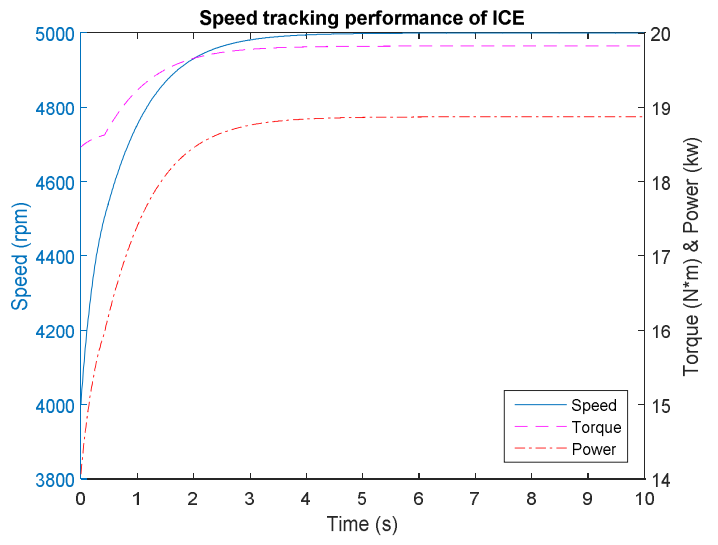

(a)

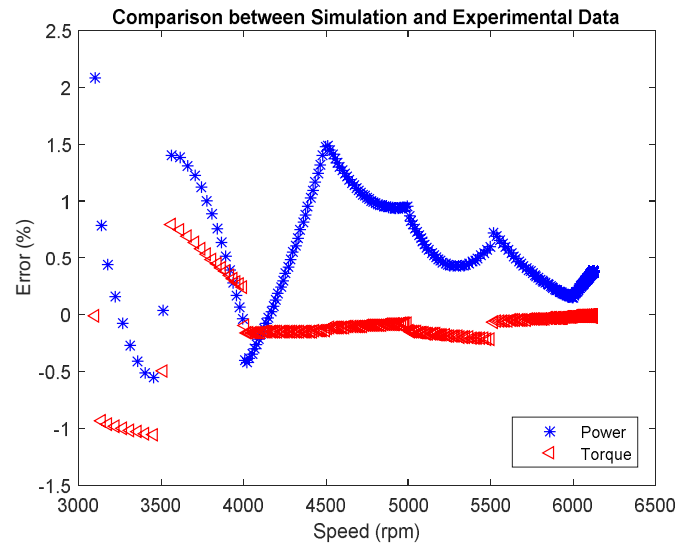

(b)

Figure 10 - Torque and power percentage errors between simulation and experimental data of the engine
Then, the approximation of steady-state characteristics of the engine model is verified by comparing the simulation data with experimental measurements. Figure $10 \mathrm{~b}$ shows the torque and power percentage error under different steady states. For the speed range considered, the calculated error is always less than $2.5 \%$. It is worth mentioning that experiments were conducted in the speed range 3000-6500 rpm to protect the engine from overheating or permanent damage.

\section{B. Simulation of Propulsion System-Test cases}

This section presents the simulation results of the entire hybrid propulsion system using two different hypothetical flight test scenarios. The first aims to verify the transition performance between different modes. The second one illustrate that the rule-based control can guarantee the engine to operate at the optimal fuel economy condition, i.e. IOL. Therefore, test case 1 only contains level-flight and climbing; while test case 2 simulates a complete flight mission that includes take-off, climbing, cruising, and landing phases.

1) Test case 1-Figure 11 and Figure 12 show the simulation results using the first hypothetical flight test scenario. The aircraft is flown using the ICE and electric motor for the first 210 seconds. Past 210 seconds, the electric motor takes over and powers the aircraft (i.e. Electric Mode). In the first phase between 0-40 sec. the Extra Charge Mode is activated following relatively low power request for flying and then again between 180-210 sec., the battery is charged up prior to transiting to the Electric Mode. The regular charging mode is activated between $60-80 \mathrm{sec}$. A short period to test the Combined Mode is performed between 120-160 sec. The Fuel Mode is in control for the rest time.
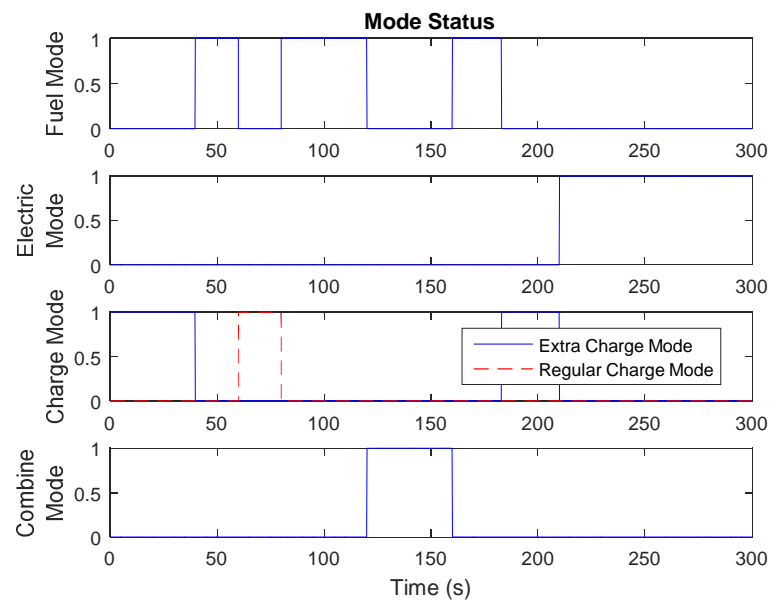

Figure 11 - Mode status of propulsion system simulation - Test case 1 
Please note that to verify the control logic and analyse the control performance, a Regular Charge Mode is activated just for a short period by increasing the value of 'charge_soc' (see Figure 8) using the external command. What's more, to enable the Fuel Mode, the condition on entering the Combined Mode is set to when power requirement is higher than the maximum engine power in this test.

As shown in top graph Figure 12, the engine and motor/generator can both follow the torque reference with a good performance with their low-level controller (speed or torque controller). However, the torque deviation occurs at the transition between Fuel Mode and Regular Charge Mode. The relatively big perturbation at Combined Mode switch is due to a significant change in the total power requirement. After being disengaged, the motor/generator continues to follow the torque requirement while the engine shaft rotates with no load, i.e. output torque is zero.

The relationship between torques in Figure 12 can provide more insight into how the system is operating. The torque requirement is the sum of engine and motor/generator torque, described as $T_{\text {req }}=G T_{i c e}+T_{\text {mot } / g e n}$. It should be noted that the value of torque, which is displayed with the blue solid line, is deduced by multiplying the torque of the engine with the gear ratio. Hence, the relationship between the components torque and torque requirements is more intuitive to verify.

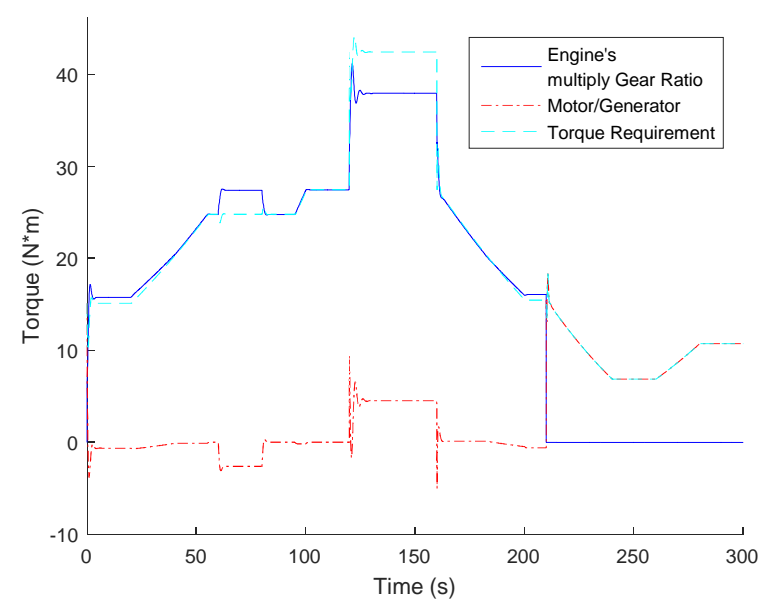

Figure 12 - Components results of propulsion system simulation - Test case 1

It can be clearly seen that $G T_{i c e}$ is higher than $T_{\text {req }}$ during $0-40 \mathrm{sec}$, and 180-210 sec, which implies that the extra power from engine is utilized to charge the battery via the generator. Whereas, the motor contributes to driving the propeller when $G T_{i c e}$ is lower than $T_{r e q}$ during the Combined Mode (the time interred between 120-160 sec). Then, the engine torque decreases to zero and the motor provides all the requested torque.
2) Test case 2-Figure 13 and Figure 14 show the simulation results of a ten minutes flight. The aircraft takeoff run is in the first $50 \mathrm{sec}$ and then continue to climb to the indicated cruise height during 50-200 sec. Past 210 seconds, the aircraft is in cruise phase for around $300 \mathrm{sec}$ and finally landing process is activated. The power requirement of the above illustrated flight mission is shown in Figure 13.

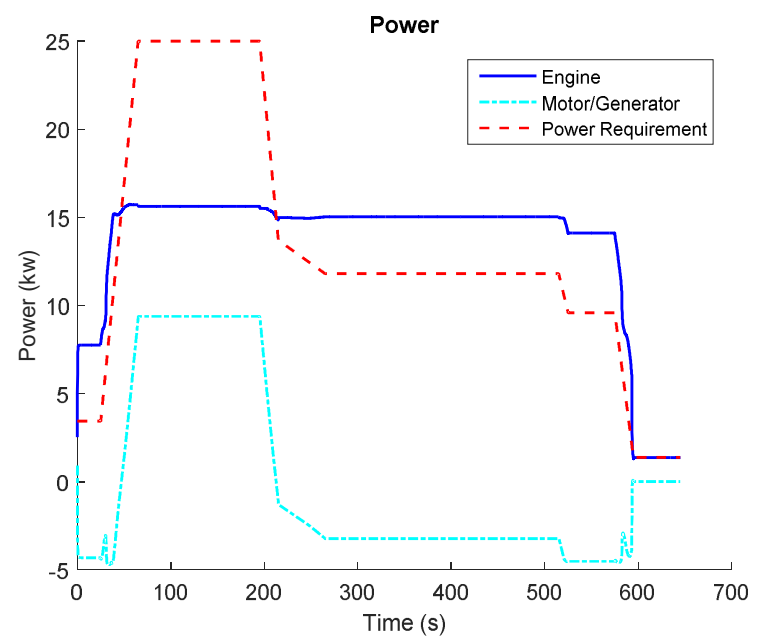

(a)
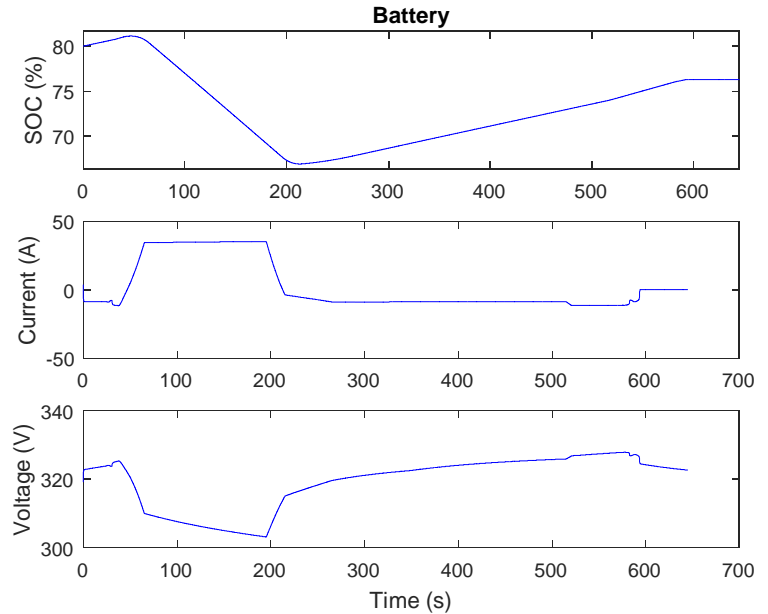

(b)

\section{Figure 13 - Components results of propulsion system simulation - Test case 2}

As opposed to the first case, in this second case the engine and motor/generator are available during the entire flight mission, i.e. the Electric Mode will not be activated. As a result, the HEPS spends most of time in Charge Mode and Combined Mode and nearly no time to enter the Fuel Mode. The supervisory controller focuses on power distribution between the engine and motor/generator. As shown in Figure $13 \mathrm{a}$, except for the take-off and landing phases, the engine 
runs at a constant power with high efficiency and this can also indirectly extend the engine life. The power from the motor is used to compensate for the difference between the engine power and demanded power requirement.

The battery results provide an insight into the battery voltage, current and SOC during this ten-minute hypothetical flight. It indicates the same operating principle as shown in the power profiles, but provides more information on charging/discharging cycle. It is already known that the extra charging power is obtained by comparing with the IOL, so the charging in the Extra Charge Mode (0-40 sec and 210$\mathrm{sec}$ ) is usually slow. Moreover, in this test case the battery charging current is much smaller than the discharging current. Given that in most flights this will be the case, it is recommended that the battery SOC should be kept high during the complete flight mission just in case of an emergency, for example in case of an engine failure.

From Figure 14, it can be concluded that the engine not only operate at near constant power (see Figure 13a), it can actually operate on the IOL. Put it differently, the designed rule-based energy management controller can ensure the minimum fuel consumption of the complete powertrain. In fact the simulation shows that for the same mission, under the same flight conditions, the aircraft equipped with the HEPS can have at least $7 \%$ better fuel economy, in comparison with an engine only powered aircraft.

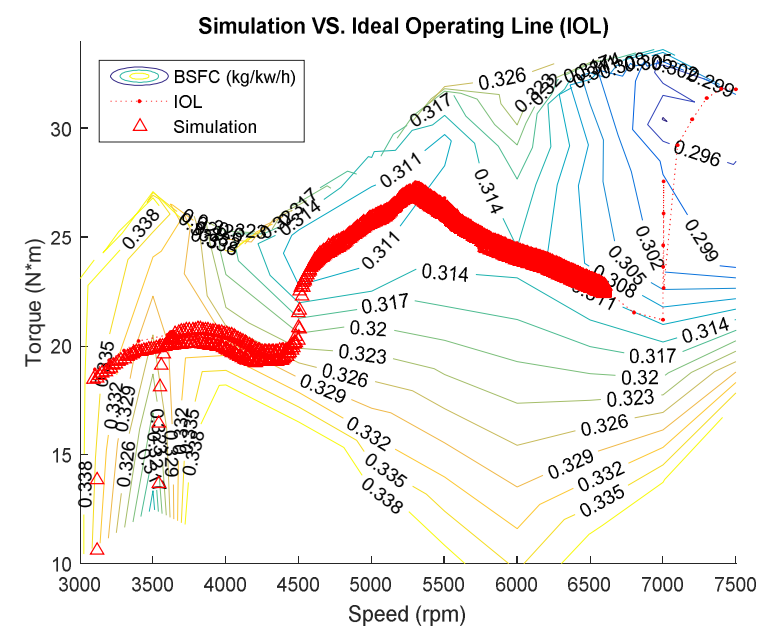

Figure 14 - Engine torque results vs. ideal operating line - Test case 2

\section{Conclusion}

This paper presented the modelling and control of a HEPS designed for UAVs. The main characteristics of the complete system were highlighted. Part of the complete parallel hybrid powertrain was simplified in order to focus on the three components that have a significant impact on the system performance namely, the battery, engine and electric motor/generator. It also made the model development and control design easier to implement.

The engine model was validated by analysing the tracking performance and comparing simulation results with experimental data. The model provided an insight in the inherent relationship between the throttle command and the torque output. One of the advantages of this model is the low computational time since extra calculations of the engine torque loss is not needed. With respect to the $d$ - $q$ model of motor/generator, the torque loss is always important to approximate the behaviour at steady state. The efficiency map was used and the torque loss function is developed, though a further research is required to obtain the algebraic representation. The reduction gear ratio (engine output shaft speed to motor/generator) is fixed at 2.2. This is to maximize the lifespan of the motor/generator.

Since the project is still at an early stage and the system is currently being developed. For the control strategy, a rulebased energy management strategy was developed on the basis of analysing the engine IOL. The transition between operating modes and components behaviours were tested in simulation using a hypothetical 300 seconds flight test case 1 , during which all operating modes of the HEPS were tested. Test case 2 validates that the designed energy management control can realize system power distribution and optimal operation. For the energy management strategy, the fuel consumption is the only optimisation criteria. Future works include the development of advanced controllers; formatting the problem as a multi-objective optimization problem and modelling of some of the subcomponents in more details.

\section{REFERENCES}

[1] Chan, C. C. "The state of the art of electric and hybrid vehicles." Proceedings of the IEEE 90.2 (2002): 247275 .

[2] Dudek, Magdalena, et al. "Hybrid fuel cell-battery system as a main power unit for small Unmanned Aerial Vehicles (UAV)." Int. J. Electrochem. Sci 8 (2013): 8442-8463.

[3] Harmon, Frederick G., Andrew A. Frank, and Sanjay S. Joshi. "The control of a parallel hybrid-electric propulsion system for a small unmanned aerial vehicle using a CMAC neural network." Neural Networks 18.5 (2005): 772-780.

[4] Ausserer, Joseph K. "Integration, testing, and validation of a small hybrid-electric remotely-piloted aircraft." Air Force Institute of Technology, WPAFB, Master's Thesis, 2012. 
[5] Michael P. Molesworth. "Rapid Prototype Development of a Remotely-Piloted Aircraft Powered by a HybridElectric Propulsion System." Air Force Institute of Technology, WPAFB, Master's Thesis, 2012.

[6] Glassock, Richard R., Jane Y. Hung, Luis F. Gonzalez, and Rodney A. Walker. "Multimodal hybrid powerplant for unmanned aerial systems (UAS) Robotics." 24th Bristol International Unmanned Air Vehicle Systems Conference, Bristol, 2009.

[7] Koster, Jean, Cody Humbargar, Eric Serani, Alex Velazco, Derek Hillery, and Les Makepeace. "Hybrid Electric Integrated Optimized System (HELIOS): Design of a Hybrid Propulsion System for Aircraft." In 49th AIAA Aerospace Sciences MEeting including the New Horizons Forum and Aerospace Exposition, p. 1011. 2011.

[8] TEL AVIV. "Bental demonstrates hybrid UAV engine. " May, 22, 2007. https://www.flightglobal.com/news/articles/bentaldemonstrates-hybrid-uavengine-213939/. [Accessed Feb. 2016]

[9] Evhangar. "Flight Design 914mh $30 \mathrm{~kW}$ electric peak power (POC)." July 26, 2010, https://www.evhangar.com/system/\%20mheps/flightdesign-914mh/. [Accessed Feb. 2016]

[10] Jason Paur. "Siemens Builds the Chevrolet Volt of Airplanes." June 23, 2011,http://www.wired.com/autopia/2011/06/electricairplane-uses-hybrid-power-similar-to-chevy-volt/. [Accessed Feb. 2016]

[11]Chiara, Fabio, Junmin Wang, Chinmaya B. Patil, MingFeng Hsieh, and Fengjun Yan. "Development and experimental validation of a control-oriented Diesel engine model for fuel consumption and brake torque predictions." Mathematical and Computer Modelling of Dynamical Systems 17.3 (2011): 261-277.

[12] Urrahman, Syed Fazal. "Modeling and Control Methods for Improving Drivability, Power Management and Fuel Economy in a Hybrid Electric Vehicle." PhD diss., Ph. D Dissertation of Wayne State University, 2008.

[13]Lin, C-C., Zoran Filipi, L. Louca, H. Peng, D. Assanis, and J. Stein. "Modelling and control of a medium-duty hybrid electric truck." International Journal of Heavy Vehicle Systems 11.3-4 (2004): 349-371.

[14] Krause , P. "Analysis of electric machinery." 1986, McGraw Hill.
[15]Wei, Xi. "Modeling and control of a hybrid electric drivetrain for optimum fuel economy, performance and driveability." PhD diss., The Ohio State University, 2004.

[16]B. M. Baumann, G. Washington, B. C. Glenn, and G. Rizzoni, "Mechatronic design and control of hybrid electric vehicles," IEEE/ASME Transactions on Mechatronics, vol. 5, no. 1, pp. 58-72, 2000.

[17] N. J. Schouten, M. A. Salman, and N. A. Kheir, "Fuzzy logic control for parallel hybrid vehicles," IEEE Transactions on Control Systems Technology, vol. 10, no. 3, pp. 460-468, 2002.

[18] L. V. Perez, G. R. Bossio, D. Moitre, and G. O. Garcia, "Optimization of power management in an hybrid electric vehicle using dynamic programming," Mathematics and Computers in Simulation, vol. 73, no. 1-4, pp. 244-254, 2006.

[19] Harmon, Frederick G. "Neural network control of a parallel hybrid-electric propulsion system for a small unmanned aerial vehicle. " PhD's Thesis, University of California Davis, 2005.

[20] Hiserote, Ryan M. "Analysis of hybrid-electric propulsion system designs for small unmanned aircraft systems. " Air Force Institute of Technology, WPAFB, Master's Thesis, 2010.

[21]Rankine, William John Macquorn. On the mechanical principles of the action of propellers. publisher not identified, 1865.

[22] Froude, R. E. "On the part played in propulsion by differences of fluid pressure." Transactions of the Institute of Naval Architects 30 (1889): 390-405.

[23] Urrahman, Syed Fazal. "Modeling and Control Methods for Improving Drivability, Power Management and Fuel Economy in a Hybrid Electric Vehicle." PhD diss., Wayne State University, 2008.

[24]Hung, J. Y., and Luis Felipe Gonzalez. "On parallel hybrid-electric propulsion system for unmanned aerial vehicles." Progress in Aerospace Sciences 51 (2012): 117.

[25]Pillay, Pragasen, and Ramu Krishnan. "Modeling, simulation, and analysis of permanent-magnet motor drives. I. The permanent-magnet synchronous motor drive." Industry Applications, IEEE Transactions on 25.2 (1989): 265-273.

[26] Pillay, Pragasan, and Ramu Krishnan. "Modeling of permanent magnet motor drives." Robotics and 
IECON'87 Conferences. International Society for Optics and Photonics, 1987.

[27] Evangelou, Simos A., and Amit Shukla. "Advances in the modelling and control of series hybrid electric vehicles." American Control Conference (ACC), 2012. IEEE, 2012.

[28] Rosado, Sebastian P. "Voltage stability and control in autonomous electric power systems with variable frequency." PhD diss., Virginia Polytechnic Institute and State University, 2007.

\section{BIOGRAPHY}

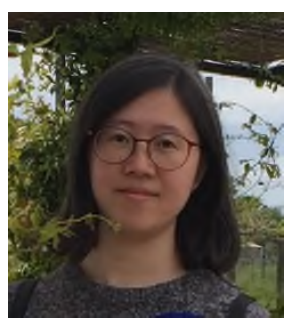

Ye Xie received a B.S. and M.S. in Automation Engineering from Nanjing University of Aeronautics and Aeronautics, China in 2012. She is a PhD Candidate in Aerospace Engineering from Cranfield University, UK. She has a background on robust control, guidance, UAV, and embedded system, with a focus on control and intelligent energy management of hybrid power for UAVs. Currently she is working on InnovateUK funded AirStart projects.

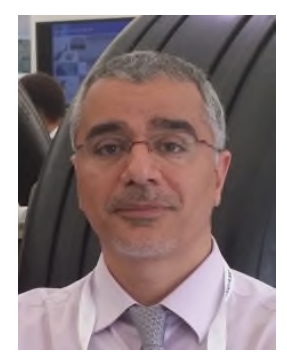

Al Savvaris is a Reader in the Centre for Cyber-Physical Systems at Cranfield University. $\mathrm{He}$ established the Autonomous Vehicle Dynamics and Control MSc course and the COMAC training programme at Cranfield. His research interests and activities include systems integration, hybrid energy management, communication systems, embedded systems, guidance and control. Currently he is working on InnovateUK funded AirStart and USMOOTH projects. In the past he worked on the FLAVIIR and ASTRAEA UAS projects, developing new technologies for unmanned systems; working on hardware and system integration. He participated in FP6, in the scope of the FLYSAFE project, working on next generation integrated safety systems. He was a member of the Autonomous Systems National Technical Committee; EPSRC College review member and reviewer on several international publications including IMechE and IEEE. He has published over 50 peer-reviewed journal and conference papers.
[29] Tremblay, Olivier, and Louis-A. Dessaint. "Experimental validation of a battery dynamic model for EV applications." World Electric Vehicle Journal 3.1 (2009): 1-10.

[30] Shepherd, Clarence M. "Design of primary and secondary cells II. An equation describing battery discharge." Journal of the Electrochemical Society 112.7 (1965): 657-664.

[31] Oudijk, M. F. "Optimization of CVT control: For hybrid and conventional drive lines." $\mathrm{PhD}$ diss., Masters Thesis, Eindhoven University of Technology, The Netherlands, 2005.

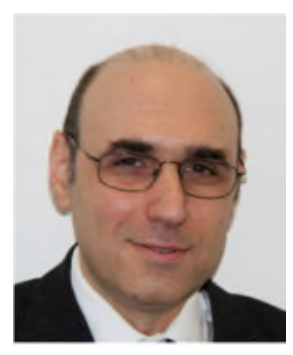

Antonios Tsourdos is a professor of control systems at Cranfield University. Appointed Head of the Autonomous Systems Group in 2007. Professor Tsourdos was member of the Team Stellar, the winning team for the UK MoD Grand Challenge (2008) and the IET Innovation Award (Category Team, 2009). Antonios is an editorial board member on several international publications including IMechE and IEEE. He is member if the IFAC Technical Committee on Intelligent Autonomous Vehicles, the IET Executive Team on Robotics and Automation and the ATI Autonomous Systems National Technical Committee. He was also involved in the SEAS DTC on Autonomous Systems Verifications. Professor Tsourdos has also been engaged in research on guidance \& control for single and multiple vehicles as well as verifiable autonomy of autonomous systems and lately dealing with the newly-important subjects of integrated system health management and cyber-physical systems. He has published over 100 peerreviewed journal and conference papers. 
2018-06-28

\section{Modelling and control of a hybrid electric propulsion system for unmanned aerial vehicles}

Xie, Ye

IEEE

Ye Xie, Al Savvaris and Antonios Tsourdos, et al., Modelling and control of a hybrid electric propulsion system for unmanned aerial vehicles. Proceedings of the 2018 IEEE Aerospace Conference, 3-10 March, Big Sky, MA, USA.

https://doi.org/10.1109/AERO.2018.8396436

Downloaded from Cranfield Library Services E-Repository 